Article

\title{
Effect Sizes, Power, and Biases in Intelligence Research: A Meta-Meta-Analysis
}

\author{
Michèle B. Nuijten 1,*俄, Marcel A. L. M. van Assen 1,2 , Hilde E. M. Augusteijn ${ }^{1}$, \\ Elise A. V. Crompvoets ${ }^{1}$ and Jelte M. Wicherts ${ }^{1}$ D \\ 1 Department of Methodology \& Statistics, Tilburg School of Social and Behavioral Sciences, \\ Tilburg University, Warandelaan 2, 5037 AB Tilburg, The Netherlands; \\ m.a.l.m.vanassen@tilburguniversity.edu (M.A.L.M.v.A.); h.e.m.augusteijn@tilburguniversity.edu (H.E.M.A.); \\ e.a.v.crompvoets@tilburguniversity.edu (E.A.V.C.); j.m.wicherts@uvt.nl (J.M.W.) \\ 2 Section Sociology, Faculty of Social and Behavioral Sciences, Utrecht University, Heidelberglaan 1, \\ 3584 CS Utrecht, The Netherlands \\ * Correspondence: m.b.nuijten@tilburguniversity.edu; Tel.: +31-13-466-2053
}

Received: 7 May 2020; Accepted: 24 September 2020; Published: 2 October 2020

\begin{abstract}
In this meta-study, we analyzed 2442 effect sizes from 131 meta-analyses in intelligence research, published from 1984 to 2014, to estimate the average effect size, median power, and evidence for bias. We found that the average effect size in intelligence research was a Pearson's correlation of 0.26 , and the median sample size was 60 . Furthermore, across primary studies, we found a median power of $11.9 \%$ to detect a small effect, $54.5 \%$ to detect a medium effect, and $93.9 \%$ to detect a large effect. We documented differences in average effect size and median estimated power between different types of intelligence studies (correlational studies, studies of group differences, experiments, toxicology, and behavior genetics). On average, across all meta-analyses (but not in every meta-analysis), we found evidence for small-study effects, potentially indicating publication bias and overestimated effects. We found no differences in small-study effects between different study types. We also found no convincing evidence for the decline effect, US effect, or citation bias across meta-analyses. We concluded that intelligence research does show signs of low power and publication bias, but that these problems seem less severe than in many other scientific fields.
\end{abstract}

Keywords: meta-meta-analysis; meta-science; bias; intelligence; power; effect size

\section{Introduction}

Mounting evidence suggests that the literature in psychology and related fields paints an overly positive picture of effects and associations. Many published findings could not be replicated in novel samples (Eerland et al. 2016; Hagger et al. 2016; Klein et al. 2014; Open Science Collaboration 2015; Wagenmakers et al. 2016), many meta-analyses highlight selective reporting of results depending on significance (Button et al. 2013; Fanelli et al. 2017; Niemeyer et al. 2012, 2013), and the number of confirmed hypotheses in the literature is incompatible with the generally small sample sizes in psychological studies (Bakker et al. 2012; Fanelli 2010; Francis 2014; Marszalek et al. 2011). It is argued that the main cause for this "replicability crisis" (Baker 2016) is a combination of publication bias and strategic use of flexibility in data analysis (Ioannidis 2005; Munafò et al. 2017). Publication bias is the phenomenon where statistically significant results have a higher probability of being published than non-significant results (Greenwald 1975). Concerning flexibility in data analysis, it is suspected that many researchers try out multiple analysis strategies to search for a significant finding, and only report the ones that "worked" (Bakker et al. 2012; John et al. 2012; Simmons et al. 2011). This increases false positive rates and generally inflates estimates of genuine effects. Because both biases might negatively 
affect the trustworthiness of published findings, it is important to assess their severity in different bodies of literature. In this paper, we investigated effect size, power, and patterns of bias in the field of intelligence research.

Intelligence research provides a good field to study effect size, power, and biases because it encompasses a wide range of study types using different methods that still focus on measures of the same construct. Intelligence is among the most well-known constructs in psychology and has been investigated extensively from various angles since the development of the first successful intelligence tests in the early 20th century (Binet and Simon 1905; Hunt 2010; Mackintosh 2011, for reviews, see, e.g., Ritchie 2015). Individual differences in intelligence and cognitive ability tests have been related to many relevant outcomes, correlates, and (potential) causes in the contexts of education, health, cognitive development and aging, economic outcomes, genes, and toxic substances (e.g., adverse effects of lead or alcohol exposure). Intelligence research is a multidisciplinary field with links to behavior genetics, educational sciences, economics, cognitive psychology, neuroscience, and developmental psychology. These different types of research use different methods and involve different effect sizes, and hence might differ in how strongly they are affected by potential biases (Ioannidis 2005). For instance, effect sizes are expected to be fairly large in research relating one type of cognitive test (e.g., fluid reasoning tasks) to another type of cognitive test (e.g., spatial ability tasks) because of the well-established phenomenon of the positive manifold (e.g., Van Der Maas et al. 2006). Conversely, research that attempts to improve intelligence by certain interventions might show smaller effects in light of longstanding challenges in raising intelligence (e.g., Spitz 1986). Similarly, some research methods in the study of intelligence are more challenging in terms of data collection (e.g., neuroscientific measures, twin designs in behavior genetics, or controlled interventions) than other research methods (e.g., studies that establish correlations between existing measures in readily accessible samples). This may create variations in sample sizes that play a key role in power and (over)estimation of effects and associations.

One way to investigate bias in science is by analyzing patterns in effect size estimates in meta-analyses (see, e.g., Fanelli and Ioannidis 2013; Fanelli et al. 2017; Jennions and Moeller 2002; Van Aert et al. 2019). A relevant example is the recent meta-meta-analysis by Pietschnig et al. (2019), in which they analyzed patterns of bias in 36 meta-analyses (comprising 1391 primary effect sizes) published in the journal Intelligence. They found that a majority of individual meta-analyses contained evidence for declining effect sizes over time but no significant, overall decline effect. Our work complements the work of Pietschnig et al. (2019) by analyzing a larger and more diverse sample of meta-analyses in intelligence research and by analyzing more patterns of bias. Specifically, we analyzed 2442 primary effect sizes from 131 meta-analyses in intelligence research, published between 1984 and 2014, to estimate the average effect size, median power, and evidence of small-study effects, decline effects, US effects, and citation bias. Our data, analysis scripts, and an online Appendix with supplemental analyses and results are available from https:/osf.io/z8emy/.

\section{Method}

\subsection{Sample}

We searched for meta-analyses about IQ and intelligence on ISI Web of Knowledge (one of the most well-known interdisciplinary databases) on 29 August 2014. Using the search string "TS = (IQ OR intelligence) AND TS = (meta-analysis)" we found 638 records. From these 638 records, we excluded six duplicate records and 21 records that were not available in English. We then looked at the abstracts of the articles and excluded a further 208 articles that did not report a quantitative meta-analysis, and we excluded another 107 meta-analyses that did not use IQ tests or other cognitive maximum performance tests that were featured in Carroll's (1993) seminal review of the intelligence literature (Carroll 1993). Finally, we excluded 28 records that were only available in print format or put behind paywalls that our library had no subscription to. The articles we could not access were diverse in terms 
of publication year, impact factor, and study type, which leads us to suspect that these excluded articles are not systematically different from the included ones. Note also that not all meta-analyses eventually offered useful data. Therefore, we do not suspect that adding the handful of meta-analyses from these excluded articles would have affected our conclusions substantially. In the end, we obtained a set of meta-analyses spanning 30 years, with primary studies published from 1915 to 2013, spanning almost a century.

All effect sizes retrieved from the meta-analyses were based on independent samples both within and between meta-analyses (below we indicate how we dealt with potential overlap between meta-analyses). Some meta-analyses reported the results from different types of cognitive tests from the same sample. If the meta-analysis reported results for Full Scale IQs (FSIQs), we included only those FSIQs. If the meta-analysis reported only Verbal IQs or Performance IQs, we selected one of these, depending on which set had the largest number of primary studies. We do note that effect sizes based on subtests rather than FSIQs will be noisier. If no IQ measure was presented in the meta-analysis, we chose the largest set that used a cognitive test (or a set of similar cognitive tests) that was strongly associated with the general factor of intelligence (McGrew 2009). Thus, whenever the meta-analysis lacked IQ measures, we included studies that used the same cognitive test (e.g., the use of Raven's Progressive Matrices Test) or highly similar tests that were labeled in the same manner in the meta-analytic article (e.g., all used fluid reasoning tasks). Because of the positive manifold between cognitive tests and fairly high correlations between broad cognitive factors (McGrew 2009), this strategy ensured inclusion of measures bearing on intelligence, while also creating less heterogeneity within meta-analyses than would have been present had we included entirely different (types of) cognitive tests.

One article contained two independent meta-analyses, so we included both. We excluded 102 meta-analyses because they did not contain sufficient data (or any data at all) to calculate the effect sizes and standard errors for the primary studies and 32 meta-analyses that used non-standard meta-analytic methods (e.g., multilevel models based on individual level data or unweighted analyses) for which we could not calculate primary effect sizes that were suitable for this meta-meta-analysis. Finally, we excluded nine meta-analyses that contained fewer than three unique primary studies, because it was not possible to estimate patterns of bias based on only two observations. The resulting sample consisted of 131 meta-analyses consisting of 2446 unique primary studies. For four primary studies, we were not able to calculate the effect size, so these were excluded from our analyses. Our final sample consisted of 2442 primary studies, with over 20 million participants in total. Figure 1 shows a schematic overview of the exclusion criteria and meta-analysis selection. A list of all included meta-analyses can be found at the end of the paper.

\subsection{Procedure}

Variables: For each meta-analysis, we coded several variables. First, we coded whether primary studies were unique in our sample, to avoid dependencies between meta-analyses. If a study appeared in more than one meta-analysis, we removed it from the meta-analysis with the most primary studies. This way, we ensured that the number of effect sizes of the individual meta-analyses would remain as large as possible.

Furthermore, for each unique primary study, we recorded the effect size that was included in the meta-analysis and its standard error (SE). Per primary study, we only included one effect size where we prioritized effects based on FSIQ, VIQ, or PIQ, or other cognitive tests, in that order (see above for details). Often, the meta-analysts calculated the effect size and its SE of a primary study themselves. Analyzing data and reporting results are error prone (see, e.g., Bakker and Wicherts 2011; Gøtzsche et al. 2007; Maassen et al. 2020; Mathes et al. 2017; Nuijten et al. 2016; Petrocelli et al. 2012). To minimize the risk of copying erroneously calculated or reported effect sizes and SEs, we calculated the effect sizes and SEs ourselves using data reported in the meta-analysis, where possible. In these cases, we did not record the effect size and SE reported by the authors. It would be an interesting 
additional study to estimate how much reported and recalculated effect sizes and SEs differed, but this was beyond the scope of this study.

Effect sizes could often be calculated with statistics such as means and standard deviations or frequency tables, and we could often calculate the SE using sample sizes or confidence intervals. If there was insufficient information available to calculate the primary studies' effect size and SE, we copied them directly from the meta-analysis. Where possible, we also recorded the primary studies' total sample size and the sample size per condition. After a first round of data collection, all effect size computations and effect size retrievals from meta-analytic articles were checked by a second coder to avoid errors and to correct any errors that emerged.

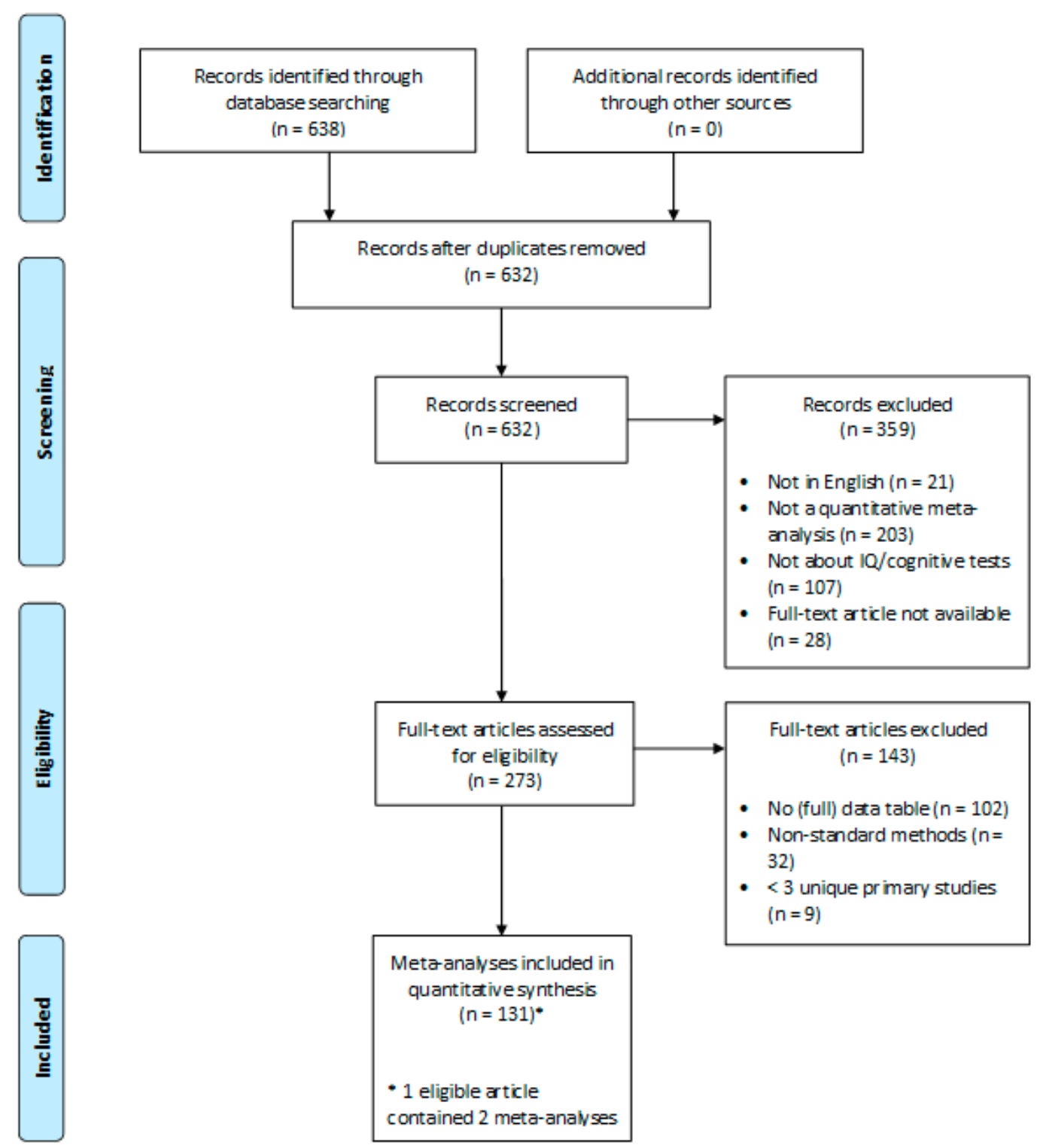

Figure 1. PRISMA Flow Diagram of the number of records identified, included and excluded, and the reasons for exclusions (Moher et al. 2009).

Finally, we categorized the meta-analyses in five different types of research: correlational, group differences, experiments and interventions, toxicology, and behavior genetics (see Table 1). We chose these five categories to distinguish between substantively different types of research questions and their associated research designs, while retaining a sufficient number of meta-analyses in each type to make reliable inferences about effect sizes, power, and bias. 
Table 1. The number of included meta-analyses and primary studies per type.

\begin{tabular}{|c|c|c|c|}
\hline Type of Research & Explanation & \# Meta-Analyses & \# Unique Primary Studies \\
\hline 1. Correlational studies & $\begin{array}{l}\text { (a) Selected IQ test is correlated with } \\
\text { other, continuous measurement of } \\
\text { psychological construct; (b) } \\
\text { test-retest correlation. }\end{array}$ & 31 & 781 \\
\hline $\begin{array}{l}\text { 2. Group differences } \\
\text { (clinical and non-clinical) }\end{array}$ & $\begin{array}{l}\text { Correlation IQ test and categorical, } \\
\text { demographical variables or clinical } \\
\text { diagnoses (e.g., male/female, } \\
\text { schizophrenia yes/no). }\end{array}$ & 59 & 1249 \\
\hline $\begin{array}{l}\text { 3. Experiments and } \\
\text { interventions }\end{array}$ & $\begin{array}{l}\text { Studies in which participants are } \\
\text { randomly assigned to conditions to } \\
\text { see if the intervention affects IQ. }\end{array}$ & 20 & 188 \\
\hline 4. Toxicology & $\begin{array}{l}\text { Studies in which IQ is correlated to } \\
\text { exposure to possibly harmful } \\
\text { substances. }\end{array}$ & 16 & 169 \\
\hline 5. Behavior genetics & Genetic analyses and twin designs. & 5 & 59 \\
\hline
\end{tabular}

Note. We categorized the meta-analyses in five different types, reflecting substantive differences in research questions and methodology, while retaining a sufficient number of meta-analyses within each type.

Correlational studies refer to studies that lack any manipulation or treatment, in which a measure of intelligence is correlated with another individual difference variable that is measured on a continuous scale. The effect sizes in such studies are typically Pearson's correlations. Examples of such studies include studies relating IQ to personality (Cohn and Westenberg 2004), brain size (McDaniel 2005), or self-rated intelligence (Freund and Kasten 2012). Studies on group differences compare existing (non-manipulated) groups and typically use Cohen's $d$ or raw mean IQ differences as the key effect size. Examples include studies comparing mean IQs between men and women (Irwing and Lynn 2005) or mean IQs between healthy controls and people diagnosed with schizophrenia (Aylward et al. 1984). Experiments and interventions are studies that attempt to improve the IQ of either healthy or unhealthy groups. Effect sizes are typically standardized mean differences, and examples include studies investigating the effect of interventions improving cognitive development in institutionalized children (Bakermans-Kranenburg et al. 2008), or the effect of iron supplementation on cognition (Falkingham et al. 2010). Studies of toxic effects on IQ entail observational studies or randomized clinical trials, in which the toxic effects relate to side effects of a certain drug treatment, often expressed in standardized mean differences. Examples include studies investigating potential harmful effects of lead exposure on IQ (Carlisle et al. 2009) or prenatal cocaine exposure on children's later IQ (Lester et al. 1998). Finally, behavior genetic studies link intelligence to genetic variations or estimate heritability using twin designs. Effect sizes are often expressed in log odds ratios or Pearson's $r$. Examples include studies about the heritability of cognitive abilities (Beaujean 2005) or studies linking specific genetic variants to general cognitive ability (Zhang et al. 2010).

We ordered the types in increasing complexity of the methodology. Correlational studies and studies about group differences usually do not require special populations and often make use of convenience samples. In experimental research, the methodology increases in complexity because participants have to be randomly assigned to carefully constructed conditions. Toxicological studies are mainly quasi-experimental but require special populations, which makes them logistically much more challenging. Finally, behavior genetic studies are arguably the most complex in methodology and often require special groups (especially in twin designs). The five study types were independently coded by $\mathrm{MN}$ and JW. The initial interrater reliability was a Cohen's $\mathrm{K}=0.868$. Any coding discrepancies were solved through discussion by the coders.

The study categorization was not defined a priori but rather decided upon ad hoc. It is possible to come up with reasonable alternative categorizations. For instance, another option would be to classify studies purely according to research design: correlational, quasi-experimental, and experimental. Effectively, this would mean combining studies on group differences and toxicological studies into one category (quasi-experimental) and distributing the meta-analyses in behavior genetics over correlational 
and quasi-experimental studies. This alternative categorization did not substantively affect the median sample sizes and effect sizes (see Section 8 in the online Appendix). However, we would like to emphasize that our categorization is one of many possibilities, and other categorizations may show different patterns in effect size, power, and bias. Our open data file allows readers to pursue other categorizations and analyses. Finally, we excluded eight primary studies that occurred in multiple meta-analyses of different types, but, after rerunning some of our descriptive analyses, we concluded that this is unlikely to have affected our conclusions (see Section 9 in the online Appendix).

Effect size conversion: For our analyses, we converted the effect sizes in all meta-analyses to a single type of effect size. For most of the meta-analyses, the effect size we extracted or calculated based on available data was either a Cohen's $d$ (80 meta-analyses; $61.1 \%$ ) or a correlation $(r ; 42$ meta-analyses; $32.1 \%$ ), so converting to one of these effect sizes seemed most convenient. We chose to convert all effect sizes to $r$ because it made more conceptual sense to express a $d$ in $r$ than vice versa. If one expresses a $d$ in $r$, the resulting point biserial correlation gives exactly the same information as $d$, but if one expresses an $r$ in $d$, the $d$ loses information (for more information, see Rosenthal and DiMatteo 2001). For the meta-analyses of patterns of bias, we subsequently converted all $r$ values to Fisher's $Z$ values, because the standard error then only depended on the sample size and not on the correlation itself (see also Sterne et al. 2005).

The direction in which the meta-analytical hypothesis was formulated can affect whether the primary effect sizes are reported as positive or negative. To correct for any influence of the direction of the hypothesis, we used a procedure called "coining," following Fanelli et al. (2017). In this procedure, we checked all meta-analytic articles that yielded a negative average meta-analytic effect and concluded that in all of these cases the result was in line with the expectations of the meta-analysts. In these cases, we multiplied all primary effect sizes within the meta-analysis by -1 . After recoding, all primary studies with negative outcomes showed an effect contradicting the hypothesis of the meta-analysts. The following analyses all used coined primary effect sizes unless stated otherwise. All our data and analysis scripts are freely available from https://osf.io/z8emy/.

\section{Effect Sizes in Intelligence Research}

We were able to convert the effect sizes from a total of 2442 primary studies to Fisher's $Z$ values. For four primary studies, we were not able to convert the effect sizes because information on sample sizes was missing. Figure 2 shows the distribution of the 2442 primary effect sizes converted back to Pearson's correlations to facilitate interpretation. The unweighted mean effect size in the 2442 primary studies was a Pearson's correlation of 0.25 ( $\mathrm{SD}=0.23$; slightly higher than the median estimate of 0.19 as observed in individual differences research; Gignac and Szodorai 2016), with a minimum of -0.94 , and a maximum of 0.95 (i.e., almost the whole range of possible values). The sample size in the primary studies varied widely, from six participants to over 1,530,000, with a median total sample size per primary study of 60 . Histograms of the correlations and total sample sizes split up per study type can be found in Section 1.2 of the online Appendix. 


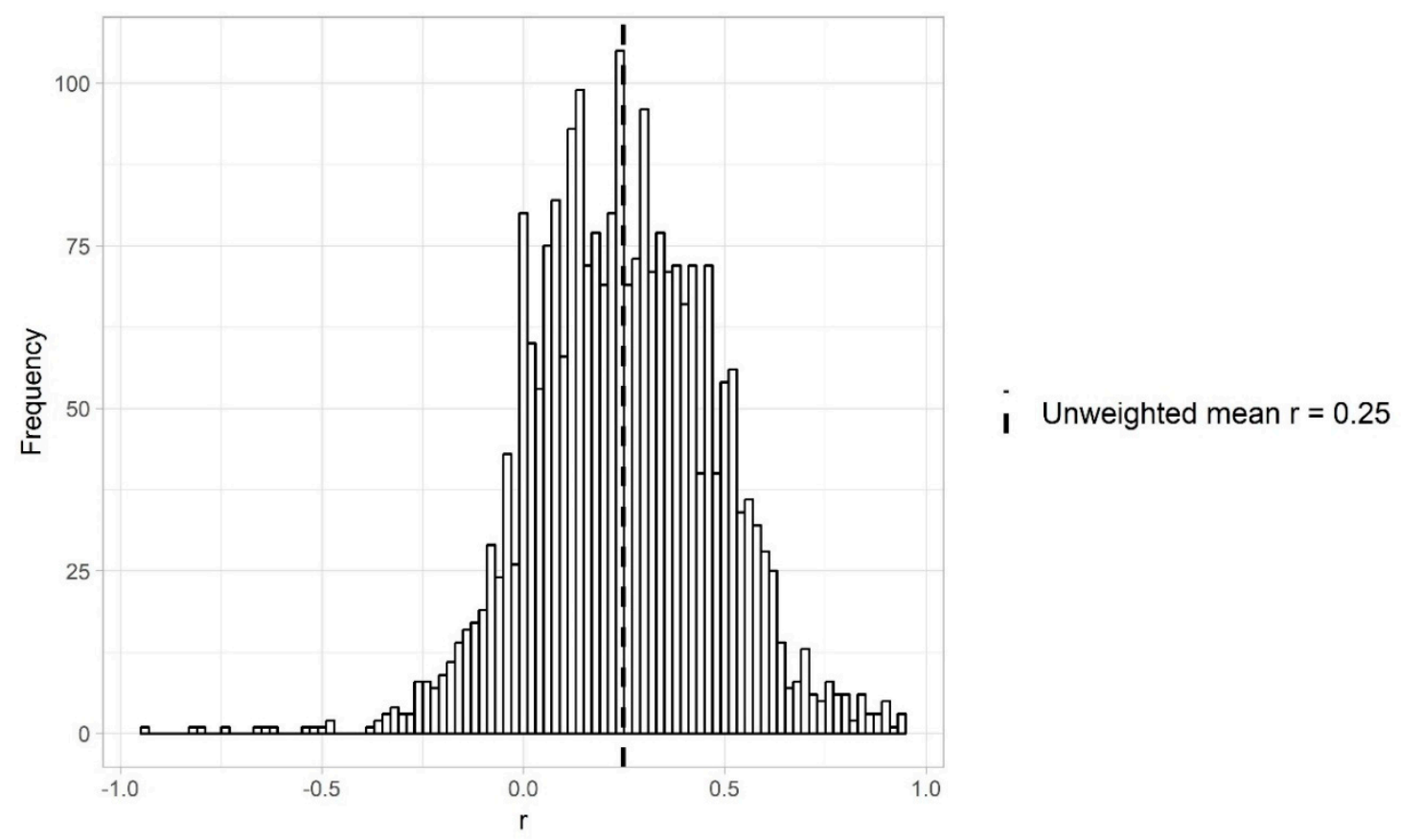

Figure 2. Histogram of the effect sizes of 2442 primary studies about intelligence. All effect sizes were converted from Fisher's Z to Pearson's correlation to facilitate interpretation.

We also looked at the sample sizes and effect sizes of the five study types separately and found some clear differences between them (see Table 2). First, the majority of meta-analyses and primary studies concerned either research about group differences in intelligence (59 meta-analyses, 1247 primary studies) or correlational research (31 meta-analyses, 779 primary studies) in which intelligence was related to other continuous psychological constructs. However, we also noted that some meta-analyses seemed to overlap substantially. For instance, in our sample we included 12 meta-analyses about the cognitive abilities in schizophrenia patients. This could be a sign of redundancy in the meta-analyses that are produced in this field, as has been found in medicine research (Ioannidis 2016).

Interestingly, in all different study types we found relatively low median sample sizes, considering the average observed effect sizes per type. This suggested that intelligence research might be generally underpowered. Note, however, that median sample sizes also varied considerably across the study types, with those of behavioral genetics $(N=169)$ being much larger than for the other four types $(\mathrm{N}=49-65)$. The meta-analytic effect size also differed across the five types. We will come back to this in the next section where we estimate the power across all meta-analyses and for the different study types separately.

\section{Power in Intelligence Research}

Several studies found that sample sizes in psychology research are typically too small to detect the expected true effect sizes (which are also estimated to be small to medium), leading to the conclusion that many psychology studies may be underpowered (Button et al. 2013; Cohen 1962; Sedlmeier and Gigerenzer 1989). Despite repeated recommendations to change this, there seems to have been no overall improvement (Fraley and Vazire 2014; Hartgerink et al. 2017; Marszalek et al. 2011; Maxwell 2004; Stanley et al. 2018; Szucs and Ioannidis 2017; Maddock and Rossi 2001; Rossi 1990; Van Aert et al. 2019). 
Table 2. Descriptive statistics of the primary studies split up into five types of studies and in total.

\begin{tabular}{|c|c|c|c|c|c|c|c|}
\hline & \# Meta-Analyses & $\begin{array}{c}\text { \# Unique } \\
\text { Primary } \\
\text { Studies }\end{array}$ & Total N & Median N & Range N & $\begin{array}{c}\text { Median } \\
\text { Unweighted } \\
\text { Pearson's } r\end{array}$ & $\begin{array}{c}\text { Median } \\
\text { Meta-Analytic } \\
\text { Effect }(r)\end{array}$ \\
\hline 1. Predictive validity and correlational studies & 31 & 779 & 367,643 & 65 & {$[7 ; 116,053]$} & 0.26 & 0.24 \\
\hline 2. Group differences (clinical and non-clinical) & 59 & 1247 & $19,757,277$ & 59 & {$[6 ; 1530,128]$} & 0.26 & 0.19 \\
\hline 3. Experiments and interventions & 20 & 188 & 24,371 & 49 & {$[10 ; 1358]$} & 0.18 & 0.17 \\
\hline 4. Toxicology & 16 & 169 & $25,720^{\mathrm{a}}$ & 60 & {$[6 ; 1333]$} & 0.15 & 0.19 \\
\hline 5. (Behavior) genetics & 5 & 59 & 30,545 & 169 & {$[12 ; 8707]$} & 0.07 & 0.08 \\
\hline Total & 131 & 2442 & $20,205,556$ & 60 & {$[6 ; 1530,128]$} & 0.24 & 0.18 \\
\hline
\end{tabular}

Note: "N" indicates number of participants in a primary study. We calculated the meta-analytic effects per subtype by taking the median of the random effects meta-analyses estimates. ${ }^{\text {a }}$

One of the meta-analyses reported two studies with non-integer total sample sizes. It seems that the authors wanted to correct their sample sizes to ensure they did not count the same

observations twice. Here, we rounded the total sample size. 
Small sample sizes and low statistical power lead to several problems. First, if a study is underpowered, the chance that it detects a true effect decreases. Second, in a set of studies containing both null effects and genuine effects, a small sample size increases the chance that a significant study represents a false positive (Ioannidis 2005). Third, when a significant finding in an underpowered study does reflect a true effect, it is likely to be overestimated (Button et al. 2013). These problems occur even when all other research practices are ideal, and there is strong evidence that they are not. Researchers have a strong focus on reporting significant results (Franco et al. 2014; LeBel et al. 2013). To obtain significant results they seem to make strategic use of flexibility in data analysis, also referred to as "researcher degrees of freedom" (Agnoli et al. 2017; John et al. 2012; but see Fiedler and Schwarz 2016; Simmons et al. 2011; Wicherts et al. 2016). Small studies are particularly vulnerable to such researcher degrees of freedom, both because they probably will not find a significant effect in the first place, but also because effect sizes are particularly strongly affected by researcher degrees of freedom in a small-study (Bakker et al. 2012).

Here, we estimated the power of the primary studies to detect a small, medium, or large effect. We retained traditional cut-offs in which small, medium, and large effects corresponded to a Pearson's $r$ of $0.1,0.3$, and 0.5 or to a Cohen's $d$ of $0.2,0.5$, and 0.8 , respectively. We calculated power using either a $t$-test for correlation (if the primary study's effect size was a correlation) or a two-sample $t$-test (if the primary study's effect size was a Cohen's $d)^{1}$, assuming $\alpha=0.05$ and two-sided tests, using the $\mathrm{R}$ package "pwr" (Champely 2017). Finally, we summarized the power estimates for all primary studies by taking their median.

Overall, we found a median power of $11.9 \%$ to detect a small effect, $54.5 \%$ to detect a medium effect, and $93.9 \%$ to detect a large effect. The power estimates differed substantially between study types (see Table 3). For example, the median power was systematically the lowest for experimental studies: $10.5 \%, 39.9 \%$, and $77.9 \%$ power to detect small, medium, and large effects, respectively. Conversely, studies in behavior genetics seemed to have the highest power of the different study types to detect small, medium, and large effects. However, it is important to note that more than half of the behavior genetics studies reported an effect smaller than what we considered a "small" effect here. This could mean that the actual power of these studies was much lower than the median power we report in Table 3.

Table 3. Median power of primary studies in intelligence research to detect a small, medium, and large effect, split up per study type and overall.

\begin{tabular}{|c|c|c|c|}
\hline \multirow{2}{*}{ Study Type } & \multicolumn{3}{|c|}{ Median Power to Detect a ... Effect * } \\
\hline & Small & Medium & Large \\
\hline 1. Predictive validity and correlational studies & $12.5 \%$ & $68.2 \%$ & $99.1 \%$ \\
\hline 2. Group differences (clinical and non-clinical) & $11.4 \%$ & $47.7 \%$ & $86.1 \%$ \\
\hline 3. Experiments and interventions & $10.5 \%$ & $39.9 \%$ & $77.9 \%$ \\
\hline 4. Toxicology & $11.9 \%$ & $47.9 \%$ & $88.2 \%$ \\
\hline 5. (Behavior) genetics & $25.1 \%$ & $92.0 \%$ & $100.0 \%$ \\
\hline Total & $11.9 \%$ & $54.5 \%$ & $93.3 \%$ \\
\hline
\end{tabular}

* Small, medium, and large effects correspond to a Pearson's $r$ of $0.1,0.3$, and 0.5 or a Cohen's $d$ of $0.2,0.5$, and 0.8 , respectively.

In order to try and approach the "real" power of each study, we also estimated the power of each primary study to detect the corresponding meta-analytic effect as a proxy for the "true" effect. Following this strategy, we found an overall median power of $51.7 \%$. Overall, less than one third

1 None of the included primary studies used a within-subjects design. 
$(30.7 \%)$ of all primary studies included in our sample reached the recommended power of $80 \%$ or higher. It is important to note that these power estimates were likely biased due to heterogeneity in the true effect sizes and noisy because of the often small number of studies included in each meta-analysis (Kenny and Judd 2019; McShane and Böckenholt 2014; McShane et al. 2020). We report details on this analysis and its results in Section 2.1 in the online Appendix.

It has been argued that estimates of the average power across an entire field lack nuance (Nord et al. 2017) and could paint the possibly misleading picture that all studies in a field are underpowered (or adequately powered) to detect a certain effect, which is not necessarily true. Indeed, across the 2442 primary studies, we found that the power of individual primary studies varied widely (see Figure 3).
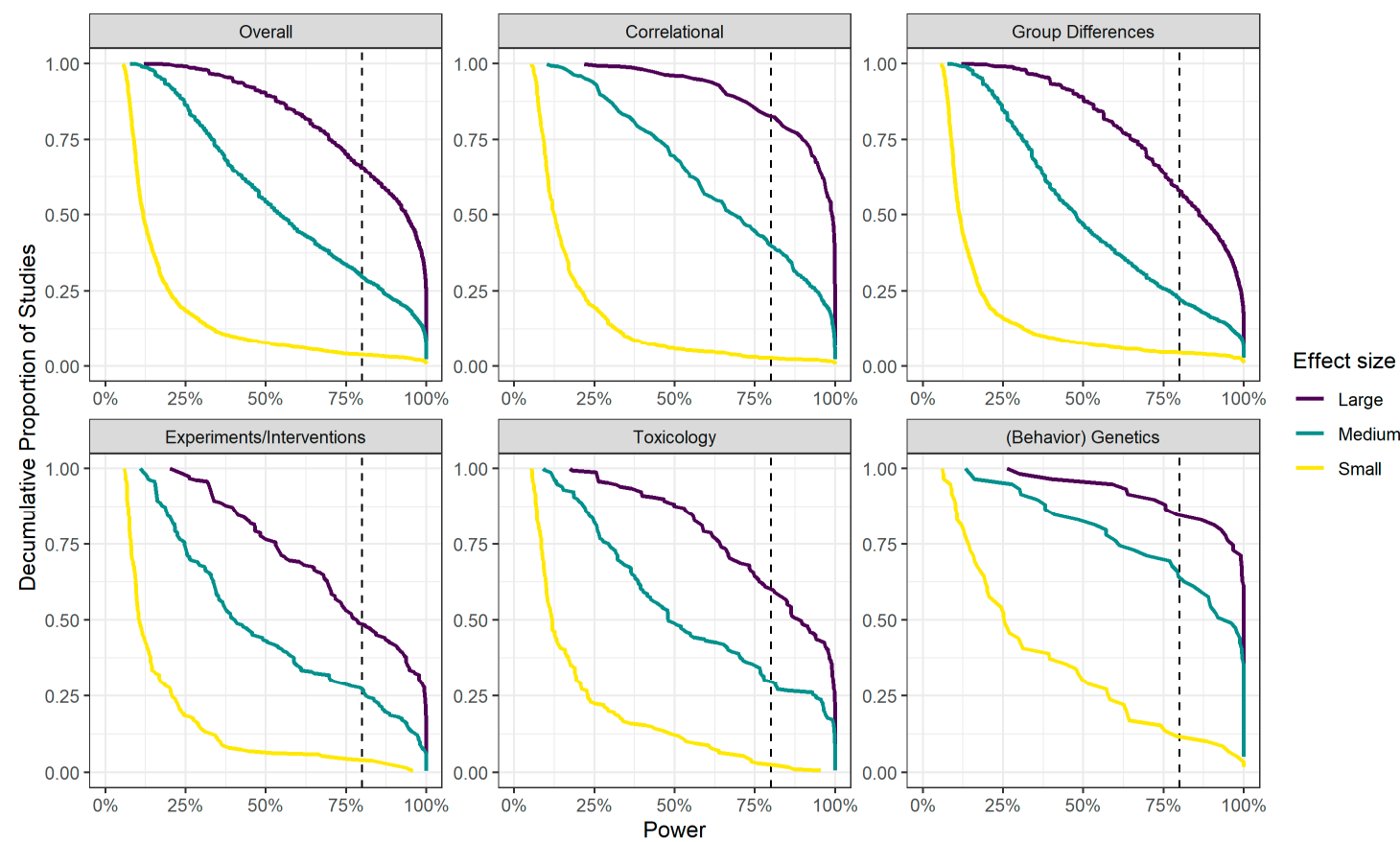

- Medium

Figure 3. Decumulative proportion of primary studies that had at least a certain power to detect a large, medium, or small effect, split up per study type and overall. The vertical dotted line indicates the nominal power of $80 \%$. Small, medium, and large effects correspond to a Pearson's $r$ of $0.1,0.3$, and 0.5 or a Cohen's $d$ of $0.2,0.5$, and 0.8 , respectively.

Figure 3 shows the decumulative proportion of primary studies that reached a certain power or more to detect a small, medium, or large effect, split up per study type and overall. The vertical dashed lines indicate a power of $80 \%$ or more. Consider, for example, the top purple line in the first panel in which the overall results are shown. The results show that only $66 \%$ of all included primary studies reached a power of $80 \%$ or more to detect a large effect (the purple line crosses the vertical line at a cumulative proportion of 0.66 ). The turquoise and yellow lines in the same panel show that $30 \%$ of the studies had sufficient power to detect a medium effect, and only $4 \%$ of the studies had sufficient power to detect a small effect.

Figure 3 also shows the distributions of power to detect different effects. For example, compare the yellow lines in the panels for group differences and behavior genetics studies. From the figure, it shows that the bulk of the studies on group differences had very low power $(<25 \%)$ to detect a small effect, represented by the drop in the curve from 1 to about 0.15 , indicating that about $85 \%$ of primary studies had very low power. Conversely, in behavior genetics studies, the bend in the curve is less pronounced, with a corresponding drop from 1 to 0.45 , indicating that about $55 \%$ of primary studies had very low power $(<25 \%)$ to detect a small effect. 
Based on these results we concluded that sample sizes in intelligence research generally seem to be too low to reliably detect hypothetical medium (or small) effects. Based on the observed effect sizes in the primary studies, it is unlikely that many effects in the intelligence literature are larger than medium. As we discussed above, small studies with low power are more at risk to overestimate effect sizes when biases are present (e.g., publication bias, or researcher degrees of freedom; Bakker et al. 2012; Nuijten et al. 2015). It is important to note that simply combining studies in meta-analyses is not sufficient to eliminate any bias from the meta-analytic estimate (Kraemer et al. 1998; Kvarven et al. 2019; Nuijten et al. 2015). If effects in primary studies are overestimated, combining them in a meta-analysis will increase precision (i.e., a smaller standard error) but result in an overall biased estimate. In the next section, we investigate whether different biases are likely to have affected effect size estimates in different types of intelligence research and in intelligence research as a whole.

\section{Bias-Related Patterns in Effect Sizes}

We investigated whether different bias-related patterns were present in intelligence research, starting with the small-study effect. A small-study effect occurs when (published) studies with smaller sample sizes yield larger average effect sizes than those with larger sample sizes (Sterne and Egger 2005). One possible cause of a small-study effect is publication bias. Smaller studies generally contain more sampling error, which means that the effect size estimates can vary widely. Effects in smaller studies need to be larger than effects in larger studies in order to reach significance thresholds. If mainly the statistically significant effects are published, small studies with overestimated effects will be overrepresented in the literature. In a meta-analysis, such a small-study effect is readily visible by verifying whether the effects in primary studies can be predicted by the studies' precision.

It is important to note that a small-study effect does not necessarily signify bias. For instance, a small-study effect can also arise because of true heterogeneity in which underlying effects happen to be related to studies' precision. For instance, in a clinical meta-analysis, the study size may be related to the intensity of the intervention: It may be the case that more strongly afflicted patients are both rare and receive more extensive treatments with larger effects than less afflicted patients who are more numerous. A small-study effect can also arise purely by chance. For an overview of alternative explanations of the small-study effect, see Sterne et al. (2005).

\subsection{Two-Step Meta-Regression}

Small-study effects can be analyzed with two-step meta-regressions (Fanelli and Ioannidis 2014; Fanelli et al. 2017; Nuijten et al. 2014). Here, bias-related patterns are investigated for each individual meta-analysis, and this information is then combined across all meta-analyses. We used this two-step strategy here. ${ }^{2}$

Within each individual meta-analysis, we estimated the small-study effect with the following meta-regression:

$$
\text { Fisher's } Z_{i j}=a^{j}+b^{j} S E_{i j}+\varepsilon_{i j}
$$

where the dependent variable Fisher's $Z_{i j}$ is the coined effect size of primary study $i$ in meta-analysis $j$ weighted by its standard error, $a^{j}$ is the intercept, $S E_{i j}$ is standard error for the primary study's effect size, and $b^{j}$ indicates the unstandardized regression coefficient of $S E_{i j}$ in predicting Fisher's $Z$. A positive $b^{j}$ coefficient would indicate that larger SEs are associated with larger effects, signaling a small-study effect. All meta-regressions were estimated in R (R Core Team 2019; version 3.6.1) using the rma()

2 It is also possible to investigate a small-study effect with multilevel weighted regression analyses that take into account that primary studies are nested within meta-analyses (Fanelli et al. 2017; Fanelli and Ioannidis 2013). A downside to this method is that it requires strong statistical assumptions that are difficult to meet with these data. For the sake of completeness, we intended to run such a multilevel analysis on our data as well, but the model was too complex for the amount of data and failed to converge. 
function in the R package metafor (Viechtbauer 2010). We assumed random effects models, and we used the Paule-Mandel estimator for random effects because it had the most favorable properties in most situations to estimate variance in true effect size between studies (Langan et al. 2017; Veroniki et al. 2016).

After running this meta-regression for each of the meta-analyses, we obtained estimates of the small-study effects (and their SEs) in the separate intelligence meta-analyses. To combine this information across meta-analyses, we then ran another meta-analysis to obtain a weighted average of all obtained regression coefficients $b^{j}$. At this meta-meta-level, we again used the Paule-Mandel estimator for random effects. We assumed random effects models at both levels, because it was highly unlikely that the same population effect underlay (1) every study within the meta-analyses, and (2) every meta-analysis' small-study effect in the meta-meta regression (Borenstein et al. 2009a). ${ }^{3}$

\subsection{Results of Small-Study Effect}

We excluded one meta-analysis from this analysis because there was too little variation in the standard errors of the primary studies to estimate a small-study effect. Across the remaining 130 meta-analyses, we found a significant overall small-study effect, $\mathrm{b}_{\mathrm{SE}}=0.67, \mathrm{SE}=0.12, Z=5.46$, $p<0.001,99 \% \mathrm{CI}=[0.35 ; 0.99], I^{2}=47.3 \%$, var (representing variation in small-study effects across meta-analyses $)=0.73(\mathrm{SE}=0.25)$. Concretely, the estimated overall small-study effect of $\mathrm{b}_{\mathrm{SE}}=0.67$ across meta-analyses meant that two otherwise identical studies with sample sizes of 25 and 100 observations would estimate Pearson's $r$ equal to $0.3(\mathrm{SE}=0.2$ ) and $0.2(\mathrm{SE}=0.1)$, respectively.

Even though we found a significant overall small-study effect across meta-analyses, this did not mean that every meta-analysis showed a small-study effect. In 17 of the individual meta-analyses $(13.1 \%)$ we found a significant small-study effect $(\alpha=0.05)$. Because this regression test has low power when meta-analyses include few studies $(\mathrm{k}<10)$, it is sometimes advised to retain a significant level of $\alpha=0.10$ (see, e.g., the example in Sterne and Egger 2005), in which case 19 meta-analyses (14.6\%) showed a significant small-study effect. We ran a robustness analysis including only meta-analyses with at least 10 primary studies and still found consistent evidence for a small-study effect (see Section 3.3 in the online Appendix). We did not find consistent differences in the small-study effect between different types of studies. See Section 3.2 in the online Appendix for details.

\subsection{Other Bias-Related Patterns}

We investigated the presence of several other bias-related patterns beside the small-study effect, following recent meta-science efforts in other fields (e.g., Fanelli et al. 2017): the decline effect, the US effect, and citation bias. A decline effect occurs when observed effect sizes decrease over time, potentially indicating overestimation in the first, more exploratory studies as compared to the more confirmatory follow-up studies (Song et al. 2010; Trikalinos and Ioannidis 2005). A US effect occurs when studies from the United States show stronger overestimation than studies from other countries, potentially caused by stronger publication pressure in the US (Doucouliagos et al. 2005; Fanelli and Ioannidis 2013; Van Dalen and Henkens 2012). Finally, citation bias occurs when larger effects are cited more often than smaller effects, which could lend unjustified importance to studies with large effects (Christensen-Szalanski and Beach 1984; Duyx et al. 2017). We explain these bias-related patterns in more detail below. Details about how we coded the relevant variables can be found in Section 1.1 of the online Appendix.

We investigated the decline effect, US effect, and citation bias using the same general two-step meta-regressions we used to estimate the small-study effect. We summarized the specific

3 In a previous discussion about estimating bias in meta-analyses (Fanelli and Ioannidis 2013; Fanelli and Ioannidis 2014; Nuijten et al. 2014) Fanelli and Ioannidis argued that choosing a random effects model at both levels unnecessarily reduces power, and they advocated the use of fixed effect models within each of the meta-analyses to decrease the amount of random fluctuation in the estimates. However, we argue that the choice for a fixed effect or random effects model is a theoretical choice, not a statistical one (Borenstein et al. 2009b). 
meta-regression equations and the results of all bias analyses in Table 4. To correct for multiple comparisons, we applied a Bonferroni correction based on the number of predictors (four main patterns of bias, including the small-study effect) for our main meta-meta-regressions (see Table 4), resulting in a significance level of 0.0125 .

Decline Effect: A decline effect occurs when studies that are published earlier in a research line report larger effects, relative to later studies. One explanation of such a decline in effect sizes is that the first study/studies of a research line are smaller and more explorative in nature, which, combined with selective publishing/reporting practices, can lead to a higher risk of overestimated effects. Subsequent studies that are larger and more confirmative in nature will likely fail to find similar extreme effects, leading to a "decline" in effect size over time. ${ }^{4}$ Previous research found evidence for decline effects in multiple scientific fields, including in intelligence research (Fanelli et al. 2017; Ioannidis 1998; Ioannidis et al. 2001; Jennions and Moeller 2002; Pietschnig et al. 2010, 2015, 2019; Song et al. 2010; Stern and Simes 1997).

We estimated the decline effect following the two-step meta-regression approach, where we used the publication order as the predictor. Specifically, if a meta-analysis contained four primary studies published in 2011, 2012, 2012, and 2013, respectively, the publication order would be 1, 2, 2, 3, respectively. We found no overall evidence for a decline effect, $b_{\text {PubOrder }}^{j}=0.001, \mathrm{SE}=0.002, \mathrm{Z}=0.371$, $p=0.711,99 \% \mathrm{CI}=[-0.003 ; 0.005], I^{2}=53.7 \%$, var $=0.00(\mathrm{SE}=0.00)$. In six cases $(4.6 \%)$ we found significant evidence for a decline effect against $\alpha=0.05$. We found no evidence that the decline effect was moderated by study type.

Our results on the decline effect are partly in line with recent results from Pietschnig et al. (2019). Using meta-meta-regressions similar to the ones we presented above, they analyzed 29 meta-analyses (comprising 991 primary effects) published in the journal Intelligence and did not find significant evidence for an overall decline effect. Pietschnig et al. (2019) performed additional analyses and concluded that a majority of individual meta-analyses did show evidence for a decline effect. We decided against interpreting patterns of bias in individual meta-analyses because individual meta-analyses often do not include sufficient studies to draw reliable conclusions about biases such as the decline effect.

In addition to the meta-meta-regressions, Pietschnig et al. (2019) investigated whether the first study in a meta-analysis is overestimated compared to subsequent studies, also known as a "winner's curse" (Ioannidis and Trikalinos 2005). To test whether such a winner's curse was present, we again used a meta-meta-regression approach in which we predicted effect size with a dummy-coded variable, indicating if a study in a meta-analysis was published first or not. We found no overall evidence for a winner's curse in our larger set of meta-analyses, $b_{\text {FirstPublished }}=0.02, \mathrm{SE}=0.01, \mathrm{Z}=1.54, p=0.123,99 \%$ $\mathrm{CI}=[-0.015 ; 0.058], I^{2}=29.8 \%$, var $=0.00(\mathrm{SE}=0.00)$.

We ran several additional analyses testing different time-related patterns in effect sizes, including the early-extremes effect (similar to the Proteus phenomenon, Ioannidis and Trikalinos 2005; also see Pietschnig et al. 2019) and several combinations of different covariates in our original analyses, but found no evidence for any systematic effects of time on effect size. We did find that across all intelligence meta-analyses, sample size seemed to increase with publication order. In other words, within a meta-analysis, studies that were published earlier had smaller samples than those published later. However, this effect was qualified by substantial heterogeneity, hence it may not generalize to all lines of intelligence research. See Section 4 in the online Appendix for details on these additional analyses.

4 For an overview of alternative explanations of a decline effect, see Trikalinos and Ioannidis 2005. 
Table 4. Overview of the meta-meta-regressions we estimated in this paper to investigate different predictors for effect size that could potentially indicate bias.

We estimated these bias-related patterns in five separate analyses.

\begin{tabular}{|c|c|c|c|c|}
\hline Type of Bias & $\begin{array}{c}\text { "Predictor" in } \\
\text { Fisher's } Z_{i j}=a^{j}+b^{j} \text { Predictor }_{i j}+\varepsilon_{i j}\end{array}$ & $\begin{array}{c}\text { Included Number of } \\
\text { Meta-Analyses }(\mathrm{m}) \text { and Number } \\
\text { of Primary Effect Sizes }(\mathbf{k})\end{array}$ & $\begin{array}{c}\text { Estimate of the Mean } \\
\text { Parameter Across } \\
\text { Meta-Analyses [99\% CI] }\end{array}$ & $\begin{array}{l}\text { Variance in the } \\
\text { Meta-Regression } \\
\text { Slopes (SE) }\end{array}$ \\
\hline 1. Small-study effect & Standard error of primary study's effect size (SE) & $\mathrm{m}=130 ; \mathrm{k}=2432$ & $0.67[0.35 ; 0.99]$ & var $=0.73(0.25)$ \\
\hline 2. Decline effect & Order of publication & $\mathrm{m}=131 ; \mathrm{k}=2442$ & $0.001[-0.003 ; 0.005]$ & var $=0.00(0.00)$ \\
\hline 3. US effect & $\mathrm{US} \times \mathrm{SE}$ & $\mathrm{m}=92 ; \mathrm{k}=2114$ & $0.46[-0.15 ; 1.06]$ & var $=0.20(0.75)$ \\
\hline 4. Citation bias & Citations per year (log transformed) & $\mathrm{m}=126 ; \mathrm{k}=2405$ & $0.008[-0.004 ; 0.020]$ & var $=0.00(0.00)$ \\
\hline
\end{tabular}


US Effect: Studies from the United States may have a higher probability of reporting overestimated effects (Fanelli and Ioannidis 2013; but see Nuijten et al. 2014). The suggested explanation for this "US effect" is that the publish-or-perish culture is stronger in the US than in other countries (Van Dalen and Henkens 2012), which would make US researchers more inclined to take advantage of flexibility in data analysis (Simmons et al. 2011) and select only (studies with) significant findings to submit for publication. To investigate whether such a US effect was present in the intelligence literature, we analyzed whether small-study effects were stronger in US studies than non-US studies (operationalized by an interaction between SE and country: US/non-US), potentially indicating stronger publication bias in the US (Doucouliagos et al. 2005; Nuijten et al. 2014).

We found a positive overall estimate of the interaction between SE and US on effect size, but this result was not statistically significant after we corrected for multiple testing (Bonferroni corrected $\alpha=0.05 / 4=0.0125), b^{j} U{ }^{*}{ }_{S E}=0.46, \mathrm{SE}=0.23, Z=1.95, p=0.051,99 \% \mathrm{CI}=[-0.15 ; 1.06], I^{2}=4.2 \%$, var $=0.20(\mathrm{SE}=0.75)$. Of the 92 meta-analyses in which we could estimate a US effect, five $(5.4 \%)$ showed significant evidence for a US effect $(\alpha=0.05)$. In our analysis of the US effect, study type was not a significant moderator. Furthermore, we also did not find evidence for a US effect when we included only meta-analyses with at least five, instead of two, (non-) US studies. See Section 5 in the online Appendix for details.

Citation Bias: Studies with larger effects may be more likely to be cited than studies with small, non-significant effects (Christensen-Szalanski and Beach 1984; Duyx et al. 2017; Jannot et al. 2013). Citation bias can cause effects to look more important or undisputed than they really are when taking into consideration all relevant evidence.

We did not find evidence for overall citation bias, $b^{j}$ CitPerYear $=0.008, \mathrm{SE}=0.005, \mathrm{Z}=1.693, p=0.091$, $99 \% \mathrm{CI}=[-0.004 ; 0.020], I^{2}=37.3 \%$, var $=0.00(\mathrm{SE}=0.00)$. We found significant evidence for citation bias in eight of the remaining 126 meta-analyses $(6.3 \% ; \alpha=0.05)$. We ran additional robustness analyses including several control variables, and consistently found no evidence for citation bias and no differences in citation bias between study types (see Section 6 in the online Appendix for details).

Robustness Checks: For all four bias patterns (small-study effect, decline effect, US effect, and citation bias) we ran several robustness checks. We reran the meta-meta-regressions, including several potential covariates (e.g., SE, sample size, journal impact factor), but none of these robustness analyses showed different overall patterns than our main analyses. We also ran an "omnibus" meta-meta-regression, in which we estimated all bias patterns at once. We did not use this omnibus test as our main analytic strategy because adding more predictors to the model lowered the power and because we could only fit the full model for 82 meta-analyses (63\%). We found patterns similar to the ones reported in Table 4. The main difference in this robustness check was that the estimate of the small-study effect was no longer significant $\left(\mathrm{bj}_{\mathrm{SE}}=0.57,99 \% \mathrm{CI}=[-0.07 ; 1.21]\right)$. The estimate of the coefficient itself, however, was similar to the one we found in the separate regression $\left(b^{j} j_{S E}=0.67\right.$, $99 \%$ CI $=[0.35 ; 0.99]$; see Table 4). Details of this "omnibus" meta-meta-regression can be found in Section 7 in the online Appendix. The additional robustness checks are reported throughout the online Appendix under the headings of the relevant bias-related patterns.

\section{Discussion}

In this study, we analyzed 2442 effect sizes from 131 meta-analyses about intelligence published between 1984 and 2014, based on over 20 million participants. We found that the median effect size in this field was a Pearson's correlation of 0.24 (unweighted mean $r=0.25$ ). This effect was similar to the typical effect in psychology $(r=0.24$; Bakker et al. 2012; based on an average total sample size of 40 and $d=0.50$ ). We found relevant differences between the subtypes of intelligence research. Specifically, we found that the types of studies that were least complex in terms of methodology were most often conducted and had the largest effect sizes; in correlational research and research about group differences we found median (unweighted) effect sizes of $r=0.26$. In less prevalent-and arguably more complex types of research—the (unweighted) effect size was lower and decreased rapidly from 
$r=0.18$ in experimental research, to $r=0.15$ in toxicological studies, and $r=0.07$ in behavior genetics. Given the typical effect sizes, the sample sizes for all study types were relatively small, with an overall median of 60 .

Across all primary studies, we found an overall median power of $11.9 \%$ to detect a small effect, $54.5 \%$ for a medium effect, and 93.9\% for a large effect (corresponding to a Pearson's $r$ of $0.1,0.3$, and 0.5 or a Cohen's $d$ of $0.2,0.5$, and 0.8 , respectively). Again, we found relevant differences between study types; power was lowest in experimental research and highest in behavior genetics, although we noted that most of the observed effect sizes in behavior genetics research were much smaller than the standard small effect (Pearson's $r=0.1$ or Cohen's $d=0.2$ ) with which we calculated power. Overall, we concluded that all study types (except for behavior genetics) generally had samples too small to reliably detect a medium effect.

We found evidence for a relatively small but statistically significant small-study effect across the intelligence literature: On average, smaller studies seemed to yield higher effect sizes, although this effect was marked by substantial variance across meta-analyses. Not all meta-analyses showed a small-study effect and some meta-analyses showed a "large study effect," i.e., larger sample sizes were associated with larger effect sizes. The presence of a small-study effect could be a sign of publication bias, especially given the overall low power we estimated. Note that even though a small-study effect is consistent with bias, it could also be explained by other factors, including true heterogeneity or chance (for an overview, see Sterne et al. 2005). All five types of studies showed consistent evidence for a small-study effect, and we did not find evidence that the small-study effect was stronger in any of these types. We found no evidence that the small-study effect was stronger for US studies (the US effect) in intelligence research. This was in line with previous findings that the US effect does not seem robust against method of analysis (Fanelli et al. 2017; Nuijten et al. 2014). We also did not find evidence for citation bias. Finally, we did not find an overall decline effect, early-extremes effect, or other time-related biases.

Compared other fields, the potential problems in intelligence research seem less severe. First, the median power in intelligence research seems higher than the median power estimated in neuroscience (8-31\%; Button et al. 2013), psychology (between 12\% and 44\%; Stanley et al. 2018, Szucs and Ioannidis 2017), behavioral ecology and animal research (13-16\% for a small effect and $40-47 \%$ for a medium effect; Jennions and Moeller 2003), economics (18\%; Ioannidis et al. 2017), and social-personality research (50\% for $r=0.20$; Fraley and Vazire 2014). Second, we did not find trends in effect sizes over time, which might indicate that the field of intelligence research is less susceptible to time-lag biases such as the decline effect or the early-extreme effect (Trikalinos and Ioannidis 2005). This was in line with the theory that such biases would affect mainly research fields in which results could be rapidly produced and published, which might not apply to the majority of studies about intelligence (Ioannidis and Trikalinos 2005). Finally, citation bias seems to be a problem in medical research (Duyx et al. 2017; Jannot et al. 2013), and there is some evidence that it also affects social sciences in general (Fanelli et al. 2017), but in intelligence research, specifically, we found no evidence that larger effects were cited more often.

\subsection{Limitations}

\subsubsection{Sample}

In our study, we were limited to meta-analyses that actually included the full data table, which was very often not the case (namely, in 81 meta-analyses). It is imaginable that the meta-analyses without data tables contained stronger and/or other patterns of bias. It could be the case that meta-analysts who go through the effort of presenting the full data in their papers are more rigorous in their work. This could then mean they may also have tried harder to find all primary studies (published and unpublished), which would have decreased overall bias in the meta-analysis. 
Furthermore, not all studies in the intelligence literature end up being included in a meta-analysis. It is also important to note that the research question of a primary study does not necessarily have to be the same as the research question of the meta-analysis. If an included primary effect size does not correspond to the main hypothesis test from that study, there is less reason to expect bias in that effect size. Conversely, if an effect is the focus of a primary study, there may be more pressure to report significant and/or inflated effect sizes. It is possible that the relatively weak bias patterns we found in this study can be explained by differences in focus in primary studies and meta-analyses.

Our sample of meta-analyses included a wide range of years of publication (1984-2014, with primary studies covering almost 100 years), but did not include meta-analyses published later than 2014. Since 2012, there has been increased attention paid to problems concerning overestimated effect sizes and low power in psychology (Open Science Collaboration 2015; Pashler and Wagenmakers 2012). As a result, biases may be weaker in more recent articles and in more recent meta-analyses, as well. However, studies in any meta-analysis normally cover many years, so even if there have been improvements in recent years, it will likely take a while before these improvements are visible in meta-analyses.

We did not have access to some meta-analyses that did match our search criteria because they were behind a paywall or not written in English. It is imaginable that there are systematic differences between these meta-analyses and the ones we did include. However, we suspect that many of these meta-analyses would have been excluded in later steps, anyway, because of overlap with other meta-analyses or missing data tables, which means that only a handful of meta-analyses would have been eligible for inclusion. Furthermore, a post-hoc analysis of this set of excluded records showed wide variation in publication year, journal impact factor, research question, and study type, so we see no immediate reason to think that this excluded sample would systematically differ from our included sample. We therefore do not expect that the exclusion of this set of meta-analyses affected our overall conclusions.

\subsubsection{Analyses}

It is important to note that we estimated many meta-regressions for meta-analyses with only few primary studies in them. This means that the individual bias estimates for each of the meta-analyses may be unreliable (Schmidt 2017). We therefore did not draw conclusions about the individual meta-analyses but summarized the obtained meta-regression coefficients in a single estimate for each of the bias patterns we investigated. It is also possible that these meta-meta-regressions are underpowered (see Fanelli and Ioannidis 2014), so any significance tests on our data need to be interpreted with care. In future research, it would be valuable to garner an even larger sample of meta-analyses, conduct formal power analyses for the (preferably preregistered) meta-meta-regressions, and consider other options for modeling the different types of bias.

When interpreting our current results, it is also important to take into account that these are patterns of potential bias that are aggregated over meta-analyses. Even though we found evidence for an overall small-study effect, this does not mean that each meta-analysis in intelligence research showed this problem. Conversely, even though we did not find evidence for an overall decline effect, US effect, or citation bias, this does not mean that these problems never occur in intelligence research. Furthermore, there are other types of scientific biases that we did not investigate here. For instance, previous studies showed evidence for a "grey literature bias" (Dickersin 2005; Fanelli et al. 2017; Glass et al. 1981; McAuley et al. 2000; Song et al. 2010). Here, unpublished literature such as Ph.D. theses or conference proceedings typically report smaller effects than research published in peer-reviewed journals, which could be a possible indicator for publication bias. Another type of bias we did not investigate is "industry bias," where sponsorship from a company may be related the size and direction of published effects (Fanelli et al. 2017; Lexchin et al. 2003). These might be interesting patterns to investigate in future research. 


\subsection{Conclusion and Recommendations}

Based on our findings, we conclude that intelligence research from 1915 to 2013 shows signs that publication bias may have caused overestimated effects. Specifically, we found that in these years power was often too low to reliably detect small or medium effects and, in general, smaller studies yielded larger effects. This is in line with the notion that publication bias and perhaps also researcher degrees of freedom in the analysis of data and reporting of results may have led to overestimated effects. Even though there might be several alternative explanations for these results, we argue that it is safe to assume that intelligence research has not been immune to the problems of robustness in psychology, although the problems in intelligence seemed to be less severe as compared to other fields.

There are several strategies to improve the reliability of primary studies and meta-analyses (Asendorpf et al. 2013; Brandt et al. 2014; Munafò et al. 2017). Some potential improvements entail top-down changes: We might need systematic changes in peer review, in allocation of funding, and in university policies. However, there are also multiple things researchers themselves can do, bottom-up (Nuijten 2018). One bottom-up strategy to improve reliability of published findings is to increase power (Button et al. 2013; Kraemer et al. 1998; Nuijten et al. 2015). Strategies to increase power include increasing sample sizes or increasing reliability of measurements. When researchers run a power analysis to determine the required sample size, researchers should think carefully about the effect size they expect to find. The standard categories of Cohen (i.e., correlations of 0.1, 0.3, and 0.5 corresponding to small, medium, and large effects, respectively) might not be realistic in every line of research (see, e.g., Gignac and Szodorai 2016 for empirical suggestions for small, medium, and large effects in individual differences research). Furthermore, researchers need to take into account that published effect sizes from previous research are probably overestimated. Ways to deal with this are correcting the observed effect sizes for publication bias (Anderson et al. 2017; Van Assen et al. 2015; Vevea and Hedges 1995), calculating lower bound power (Perugini et al. 2014), or basing a power analysis on the smallest effect size of interest (Ellis 2010). Another improvement would be to avoid publication bias and opportunistic use of flexibility in data analysis by preregistering study plans (Chambers and Munafo 2013; Wagenmakers et al. 2012). A final recommendation is to increase transparency by sharing materials, data, and analysis scripts to facilitate replication and reanalysis (Nosek and Bar-Anan 2012; Nuijten 2017; Wicherts 2013). This list of recommendations is far from exhaustive: over the years, many solutions have been suggested, targeting all steps in the empirical cycle. We feel optimistic that many of these strategies will help improve our research practices and scientific output.

Supplementary Materials: All the data, analysis scripts, and an online Appendix with supplemental analyses and results can be found on this paper's project page on the Open Science Framework at https://osf.io/z8emy/.

Author Contributions: Contributed to conception and design: J.M.W., M.B.N. Contributed to acquisition of data: H.E.M.A., E.A.V.C., J.M.W., M.B.N. Contributed to analysis and interpretation of data: M.B.N., J.M.W., M.A.L.M.v.A. Drafted and/or revised the article: M.B.N., J.M.W., M.A.L.M.v.A. Approved the submitted version for publication: M.B.N., M.A.L.M.v.A., H.E.M.A., E.A.V.C., J.M.W. All authors have read and agreed to the published version of the manuscript.

Funding: The preparation of this article was supported by VIDI grant (no. 452-11-004) from the Netherlands Organisation for Scientific Research (NWO) and ERC consolidator grant IMPROVE (grant no. 726361) from the European Research Council.

Acknowledgments: Multiple people have commented on this paper in its various stages. We would like to thank all for their insightful suggestions.

Conflicts of Interest: The sponsors had no role in the design, execution, interpretation, or writing of the study. 


\section{References}

Agnoli, Franca, Jelte M. Wicherts, Coosje L. S. Veldkamp, Paolo Albiero, and Roberto Cubelli. 2017. Questionable research practices among Italian research psychologists. PLoS ONE 12: 1-17. [CrossRef] [PubMed]

Anderson, Samantha F., Ken Kelley, and Scott E. Maxwell. 2017. Sample-size planning for more accurate statistical power: A method adjusting sample effect sizes for publication bias and uncertainty. Psychological Science 28: 1547-62. [CrossRef] [PubMed]

Asendorpf, Jens B., Mark Conner, Filip De Fruyt, Jan De Houwer, Jaap J. A. Denissen, Klaus Fiedler, Susann Fiedler, David C. Funder, Reinhold Kliegl, Brian A. Nosek, and et al. 2013. Recommendations for increasing replicability in psychology. European Journal of Personality 27: 108-19. [CrossRef]

Aylward, Elizabeth, Elaine Walker, and Barbara Bettes. 1984. Intelligence in schizophrenia: Meta-analysis of the research. Schizophrenia Bulletin 10: 430-59. [CrossRef]

Baker, Monya. 2016. 1500 scientists lift the lid on reproducibility. Nature News 533: 452. [CrossRef]

Bakermans-Kranenburg, Marian J., Marinus H. van IJzendoorn, and Femmie Juffer. 2008. Earlier is better: A meta-analysis of 70 years of intervention improving cognitive development in institutionalized children. Monographs of the Society for Research in Child Development 73: 279-93. [CrossRef]

Bakker, Marjan, and Jelte M. Wicherts. 2011. The (mis)reporting of statistical results in psychology journals. Behavior Research Methods 43: 666-78. [CrossRef]

Bakker, Marjan, Annette van Dijk, and Jelte M. Wicherts. 2012. The rules of the game called psychological science. Perspectives on Psychological Science 7: 543-54. [CrossRef]

Beaujean, A. Alexander. 2005. Heritability of cognitive abilities as measured by mental chronometric tasks: A meta-analysis. Intelligence 33: 187-201. [CrossRef]

Binet, Alfred, and Th Simon. 1905. New methods for the diagnosis of the intellectual level of subnormals. L'annee Psychologique 12: 191-244.

Borenstein, Michael, L. V. Hedges, J. P. T. Higgins, and H. R. Rothstein. 2009a. Random-effects model. In Introduction to Meta-Analysis. Edited by Michael Borenstein, L. V. Hedges, J. P. T. Higgins and H. R. Rothstein. New York: Wiley, pp. 69-76.

Borenstein, Michael, L. V. Hedges, J. P. T. Higgins, and H. R. Rothstein. 2009b. Fixed-effect versus random-effects models. In Introduction to Meta-Analysis. Edited by Michael Borenstein, L. V. Hedges, J. P. T. Higgins and H. R. Rothstein. New York: Wiley, pp. 77-86.

Brandt, Mark J., Hans IJzerman, Ap Dijksterhuis, Frank J. Farach, Jason Geller, Roger Giner-Sorolla, James A. Grange, Marco Perugini, Jeffrey R. Spies, and Anna Van't Veer. 2014. The replication recipe: What makes for a convincing replication? Journal of Experimental Social Psychology 50: 217-24. [CrossRef]

Button, Katherine S., John P. A. Ioannidis, Claire Mokrysz, Brian A. Nosek, Jonathan Flint, Emma S. J. Robinson, and Marcus R. Munafo. 2013. Power failure: Why small sample size undermines the reliability of neuroscience. Nature Reviews Neuroscience 14: 1-12. [CrossRef]

Carlisle, James C., Kathryn C. Dowling, David M. Siegel, and George V. Alexeeff. 2009. A blood lead benchmark for assessing risks from childhood lead exposure. Journal of Environmental Science and Health Part a-Toxic/Hazardous Substances E Environmental Engineering 44: 1200-08. [CrossRef]

Carroll, John B. 1993. Human Cognitive Abilities: A Survey of Factor-Analytic Studies. New York: Cambridge University Press.

Chambers, Chris, and Marcus Munafo. 2013. Trust in Science Would Be Improved by Study Pre-Registration. Available online: http://www.theguardian.com/science/blog/2013/jun/05/trust-in-sciencestudy-pre-registration (accessed on 25 September 2020).

Champely, Stephane. 2017. Pwr: Basic Functions for Power Analysis. R Package Version 1.2-1. Available online: https://CRAN.R-project.org/package=pwr (accessed on 25 September 2020).

Christensen-Szalanski, Jay J. J., and Lee Roy Beach. 1984. The citation bias: Fad and fashion in the judgment and decision literature. American Psychologist 39: 75-78. [CrossRef]

Cohen, Jacob. 1962. The statistical power of abnormal-social psychological research: A review. The Journal of Abnormal and Social Psychology 65: 145-53. [CrossRef]

Cohn, Lawrence D., and P. Michiel Westenberg. 2004. Intelligence and maturity: Meta-analytic evidence for the incremental and discriminant validity of Loevinger's measure of ego development. Journal of Personality and Social Psychology 86: 760-72. [CrossRef] 
Dickersin, Kay. 2005. Publication bias: Recognizing the problem, understanding its origins and scope, and preventing harm. In Publication Bias in Meta-Analysis: Prevention, Assessment and Adjustments. Edited by Hannah R. Rothstein, Alexander J Sutton and Michael Borenstein. New York: Wiley, pp. 11-33.

Doucouliagos, Hristos, Patrice Laroche, and Tom D. Stanley. 2005. Publication bias in union-productivity research? Relations Industrielles/Industrial Relations 60: 320-47. [CrossRef]

Duyx, Bram, Miriam J. E. Urlings, Gerard M. H. Swaen, Lex M. Bouter, and Maurice P. Zeegers. 2017. Scientific citations favor positive results: A systematic review and meta-analysis. Journal of Clinical Epidemiology 88: 92-101. [CrossRef]

Eerland, Anita, Andrew M. Sherrill, Joseph P. Magliano, Rolf A. Zwaan, J. D. Arnal, Philip Aucoin, Stephanie A. Berger, A. R. Birt, Nicole Capezza, and Marianna Carlucci. 2016. Registered replication report: Hart \& Albarracín (2011). Perspectives on Psychological Science 11: 158-71. [CrossRef]

Ellis, Paul D. 2010. The Essential Guide to Effect Sizes: Statistical Power, Meta-Analysis, and the Interpretation of Research Results. New York: Cambridge University Press.

Falkingham, Martin, Asmaa Abdelhamid, Peter Curtis, Susan Fairweather-Tait, Louise Dye, and Lee Hooper. 2010. The effects of oral iron supplementation on cognition in older children and adults: A systematic review and meta-analysis. Nutrition Journal 9: 4. [CrossRef]

Fanelli, Daniele. 2010. "Positive" results increase down the hierarchy of the sciences. PLoS ONE 5: e10068. [CrossRef] [PubMed]

Fanelli, Daniele, and John P. A. Ioannidis. 2013. US studies may overestimate effect sizes in softer research. Proceedings of the National Academy of Sciences of the United States of America 110: 15031-36. [CrossRef] [PubMed]

Fanelli, Daniele, and John P. A. Ioannidis. 2014. Reanalyses actually confirm that US studies overestimate effects in softer research. Proceedings of the National Academy of Sciences the United States of America 1117: E714-15. [CrossRef]

Fanelli, Daniele, Rodrigo Costas, and John P. A. Ioannidis. 2017. Meta-assessment of bias in science. Proceedings of the National Academy of Sciences the United States of America 114: 3714-19. [CrossRef]

Fiedler, Klaus, and Norbert Schwarz. 2016. Questionable research practices revisited. Social Psychological and Personality Science 7: 45-52. [CrossRef]

Fraley, R. Chris, and Simine Vazire. 2014. The N-pact factor: Evaluating the quality of empirical journals with respect to sample size and statistical power. PLoS ONE 9: e109019. [CrossRef]

Francis, Gregory. 2014. The frequency of excess success for articles in psychological science. Psychonomic Bulletin E Review 21: 1180-87. [CrossRef]

Franco, Annie, Neil Malhotra, and Gabor Simonovits. 2014. Publication bias in the social sciences: Unlocking the file drawer. Science 345: 1502-05. [CrossRef]

Freund, Philipp Alexander, and Nadine Kasten. 2012. How smart do you think you are? A meta-analysis on the validity of self-estimates of cognitive ability. Psychological Bulletin 138: 296-321. [CrossRef]

Gignac, Gilles E., and Eva T. Szodorai. 2016. Effect size guidelines for individual differences researchers. Personality and Individual Differences 102: 74-78. [CrossRef]

Glass, Gene V., Mary Lee Smith, and Barry McGaw. 1981. Meta-Analysis in Social Research. Beverly Hills: Sage Publications, Incorporated.

Gøtzsche, Peter C., Asbjørn Hróbjartsson, Katja Marić, and Britta Tendal Tendal. 2007. Data extraction errors in meta-analyses that use standardized mean differences. Journal of the American Medical Association 298: 430-37. [CrossRef] [PubMed]

Greenwald, Anthony G. 1975. Consequences of prejudice against the null hypothesis. Psychological Bulletin 82: 1-20. [CrossRef]

Hagger, Martin S., Nikos L. D. Chatzisarantis, Hugo Alberts, Calvin Octavianus Anggono, Cédric Batailler, Angela R. Birt, Ralf Brand, Mark J. Brandt, Gene Brewer, Sabrina Bruyneel, and et al. 2016. A multilab preregistered replication of the ego-depletion effect. Perspectives on Psychological Science 11: 546-73. [CrossRef]

Hartgerink, Chris H. J., Jelte M. Wicherts, and M. A. L. M. Van Assen. 2017. Too good to be false: Non-significant results revisited. Collabra: Psychology 3: 1-18. [CrossRef]

Hunt, Earl. 2010. Human intelligence. New York: Cambridge University Press.

Ioannidis, John P. A. 1998. Effect of the statistical significance of results on the time to completion and publication of randomized efficacy trials. Journal of the American Medical Association 279: 281-86. [CrossRef] [PubMed] 
Ioannidis, John P. A., Evangelia E. Ntzani, Thomas A. Trikalinos, and Despina G. Contopoulos-Ioannidis. 2001. Replication validity of genetic association studies. Nature Genetics 29: 306-9. [CrossRef]

Ioannidis, John P. A. 2005. Why most published research findings are false. PLoS Medicine 2: e124. [CrossRef]

Ioannidis, John P. A., and Thomas A. Trikalinos. 2005. Early extreme contradictory estimates may appear in published research: The Proteus phenomenon in molecular genetics research and randomized trials. Journal of Clinical Epidemiology 58: 543-49. [CrossRef]

Ioannidis, John P. A. 2016. The mass production of redundant, misleading, and conflicted systematic reviews and meta-analyses. The Milbank Quarterly 94: 485-514. [CrossRef]

Ioannidis, John P. A., Tom D. Stanley, and Hristos Doucouliagos. 2017. The power of bias in economics research. The Economic Journal 127: F236-F265. [CrossRef]

Irwing, Paul, and Richard Lynn. 2005. Sex differences in means and variability on the progressive matrices in university students: A meta-analysis. British Journal of Psychology 96: 505-24. [CrossRef] [PubMed]

Jannot, Anne-Sophie, Thomas Agoritsas, Angèle Gayet-Ageron, and Thomas V. Perneger. 2013. Citation bias favoring statistically significant studies was present in medical research. Journal of Clinical Epidemiology 66: 296-301. [CrossRef] [PubMed]

Jennions, Michael D., and Anders P. Moeller. 2002. Publication bias in ecology and evolution: An empirical assessment using the trim and fill method. Biological Reviews 77: 211-22. [CrossRef] [PubMed]

Jennions, Michael D., and Anders P. Moeller. 2003. A survey of the statistical power of research in behavioral ecology and animal behavior. Behavioral Ecology 14: 438-45. [CrossRef]

John, Leslie K., George Loewenstein, and Drazen Prelec. 2012. Measuring the prevalence of questionable research practices with incentives for truth-telling. Psychological Science 23: 524-32. [CrossRef]

Kenny, David A., and Charles M. Judd. 2019. The unappreciated heterogeneity of effect sizes: Implications for power, precision, planning of research, and replication. Psychological Methods 24: 578. [CrossRef]

Klein, Richard A., Kate A. Ratliff, Michelangelo Vianello, Reginald B. Adams Jr., Štěpán Bahník, Michael J. Bernstein, Konrad Bocian, Mark J. Brandt, Beach Brooks, Claudia Chloe Brumbaugh, and et al. 2014. Investigating variation in replicability: A “Many Labs" Replication Project. Social Psychology 45: 142-52. [CrossRef]

Kraemer, Helena Chmura, Christopher Gardner, John O. Brooks III, and Jerome A. Yesavage. 1998. Advantages of excluding underpowered studies in meta-analysis: Inclusionist versus exclusionist viewpoints. Psychological Methods 3: 23-31. [CrossRef]

Kvarven, Amanda, Eirik Strømland, and Magnus Johannesson. 2019. Comparing meta-analyses and preregistered multiple-laboratory replication projects. Nature Human Behaviour, 1-12. [CrossRef]

Langan, Dean, Julian Higgins, and Mark Simmonds. 2017. Comparative performance of heterogeneity variance estimators in meta-analysis: A review of simulation studies. Research Synthesis Methods 8: 181-98. [CrossRef]

LeBel, Etienne P., Denny Borsboom, Roger Giner-Sorolla, Fred Hasselman, Kurt R. Peters, Kate A. Ratliff, and Colin Tucker Smith. 2013. PsychDisclosure.Org: Grassroots support for reforming reporting standards in psychology. Perspectives on Psychological Science 8: 424-32. [CrossRef]

Lester, Barry M., Linda L. LaGasse, and Ronald Seifer. 1998. Cocaine exposure and children: The meaning of subtle effects. Science 282: 633-34. [CrossRef] [PubMed]

Lexchin, Joel, Lisa A. Bero, Benjamin Djulbegovic, and Otavio Clark. 2003. Pharmaceutical industry sponsorship and research outcome and quality: Systematic review. British Medical Journal 326: 1167-70. [CrossRef] [PubMed]

Maassen, Esther, Marcel ALM van Assen, Michèle B. Nuijten, Anton Olsson-Collentine, and Jelte M. Wicherts. 2020. Reproducibility of individual effect sizes in meta-analyses in psychology. PLoS ONE 15: e0233107. [CrossRef]

Mackintosh, Nicholas John. 2011. IQ and Human Intelligence. New York: Oxford University Press.

Maddock, Jason E., and Joseph S. Rossi. 2001. Statistical power of articles published in three health-psychology related journals. Health Psychology 20: 76-78. [CrossRef] [PubMed]

Marszalek, Jacob M., Carolyn Barber, Julie Kohlhart, and B. Holmes Cooper. 2011. Sample size in psychological research over the past 30 years. Perceptual and Motor Skills 112: 331-48. [CrossRef] [PubMed]

Mathes, Tim, Pauline Klaßen, and Dawid Pieper. 2017. Frequency of data extraction errors and methods to increase data extraction quality: A methodological review. BMC Medical Research Methodology 17: 152. [CrossRef]

Maxwell, Scott E. 2004. The persistence of underpowered studies in psychological research: Causes, consequences, and remedies. Psychological Methods 9: 147-63. [CrossRef] 
McAuley, Laura, Peter Tugwell, and David Moher. 2000. Does the inclusion of grey literature influence estimates of intervention effectiveness reported in meta-analyses? The Lancet 356: 1228-31. [CrossRef]

McDaniel, Michael A. 2005. Big-brained people are smarter: A meta-analysis of the relationship between in vivo brain volume and intelligence. Intelligence 33: 337-46. [CrossRef]

McGrew, Kevin S. 2009. CHC theory and the human cognitive abilities project: Standing on the shoulders of the giants of psychometric intelligence research. Intelligence 37: 1-10. [CrossRef]

McShane, Blakeley B., and Ulf Böckenholt. 2014. You Cannot Step Into the Same River Twice:When Power Analyses Are Optimistic. Perspectives on Psychological Science 9: 612-25. [CrossRef] [PubMed]

McShane, Blakeley B., Ulf Böckenholt, and Karsten T. Hansen. 2020. Average power: A cautionary note. Advances in Methods and Practices in Psychological Science, 1-15. [CrossRef]

Moher, David, Alessandro Liberati, Jennifer Tetzlaff, Douglas G. Altman, and The Prisma Group. 2009. Preferred reporting items for systematic reviews and meta-analyses: The prisma statement. PLoS Medicine 6: e1000097. [CrossRef] [PubMed]

Munafò, Marcus R., Brian A. Nosek, Dorothy V.M. Bishop, Katherine S. Button, Christopher D. Chambers, Nathalie Percie du Sert, Uri Simonsohn, Eric-Jan Wagenmakers, Jennifer J. Ware, and John P.A. Ioannidis. 2017. A manifesto for reproducible science. Nature Human Behaviour 1: 0021. [CrossRef]

Niemeyer, Helen, Jochen Musch, and Reinhard Pietrowsky. 2012. Publication bias in meta-analyses of the efficacy of psychotherapeutic interventions for schizophrenia. Schizophrenia Research 138: 103-12. [CrossRef]

Niemeyer, Helen, Jochen Musch, and Reinhard Pietrowsky. 2013. Publication bias in meta-analyses of the efficacy of psychotherapeutic interventions for depression. Journal of Consulting and Clinical Psychology 81: 58-74. [CrossRef]

Nord, Camilla L., Vincent Valton, John Wood, and Jonathan P. Roiser. 2017. Power-up: A reanalysis of power failure in neuroscience using mixture modeling. The Journal of Neuroscience 37: 8051-61. [CrossRef]

Nosek, Brian A., and Yoav Bar-Anan. 2012. Scientific utopia: I. Opening scientific communication. Psychological Inquiry 23: 217-43. [CrossRef]

Nuijten, Michèle B., Marcel ALM van Assen, Robbie CM van Aert, and Jelte M. Wicherts. 2014. Standard analyses fail to show that US studies overestimate effect sizes in softer research. Proceedings of the National Academy of Sciences 111: E712-E713. [CrossRef]

Nuijten, Michèle B., Marcel A.L.M. van Assen, Coosje L.S. Veldkamp, and Jelte M. Wicherts. 2015. The replication paradox: Combining studies can decrease accuracy of effect size estimates. Review of General Psychology 19: 172-82. [CrossRef]

Nuijten, Michèle B., Chris H.J. Hartgerink, Marcel A.L.M. van Assen, Sacha Epskamp, and Jelte M. Wicherts. 2016. The prevalence of statistical reporting errors in psychology (1985-2013). Behavior Research Methods 48: 1205-26. [CrossRef] [PubMed]

Nuijten, Michèle. 2017. Share analysis plans and results. Nature 551: 559. [CrossRef]

Nuijten, Michèle B. 2018. Practical tools and strategies for researchers to increase replicability. Developmental Medicine \& Child Neurology 61: 535-39. [CrossRef]

Open Science Collaboration. 2015. Estimating the reproducibility of psychological science. Science 349: aac4716. [CrossRef]

Pashler, Harold, and Eric-Jan Wagenmakers. 2012. Editors' introduction to the special section on replicability in psychological science a crisis of confidence? Perspectives on Psychological Science 7: 528-30. [CrossRef]

Perugini, Marco, Marcello Galucci, and Giulio Constantini. 2014. Safeguard power as a protection against imprecise power estimates. Perspectives on Psychological Science 9: 319-32. [CrossRef]

Petrocelli, John, Joshua Clarkson, Melanie Whitmire, and Paul Moon. 2012. When $a b \neq c-c^{\prime}$ : Published errors in the reports of single-mediator models: Published errors in the reports of single-mediator models. Behavior Research Methods 45: 595-601. [CrossRef]

Pietschnig, Jakob, Martin Voracek, and Anton K. Formann. 2010. Mozart effect-Shmozart effect: A meta-analysis. Intelligence 38: 314-23. [CrossRef]

Pietschnig, Jakob, Lars Penke, Jelte M. Wicherts, Michael Zeiler, and Martin Voracek. 2015. Meta-analysis of associations between human brain volume and intelligence differences: How strong are they and what do they mean? Neuroscience \& Biobehavioral Reviews 57: 411-32. [CrossRef] 
Pietschnig, Jakob, Magdalena Siegel, Junia Sophia Nur Eder, and Georg Gittler. 2019. Effect declines are systematic, strong, and ubiquitous: A meta-meta-analysis of the decline effect in intelligence research. Frontiers in Psychology 10: 2874. [CrossRef] [PubMed]

R Core Team. 2019. R: A Language and Environment for Statistical Computing. Available online: https: //www.R-project.org/ (accessed on 25 September 2020).

Ritchie, Stuart. 2015. Intelligence: All that Matters. London: Hodder \& Stoughton.

Rosenthal, Robert, and M. Robin DiMatteo. 2001. Meta-analysis: Recent developments in quantitative methods for literature reviews. Annual Review of Psychology 52: 59-82. [CrossRef] [PubMed]

Rossi, Joseph S. 1990. Statistical power of psychological research: What have we gained in 20 years? Journal of Consulting and Clinical Psychology 58: 646-56. [CrossRef] [PubMed]

Schmidt, Frank L. 2017. Statistical and measurement pitfalls in the use of meta-regression in meta-analysis. Career Development International 22: 469-76. [CrossRef]

Sedlmeier, Peter, and Gerd Gigerenzer. 1989. Do studies of statistical power have an effect on the power of studies? Psychological Bulletin 105: 309-16. [CrossRef]

Simmons, Joseph P., Leif D. Nelson, and Uri Simonsohn. 2011. False-positive psychology: Undisclosed flexibility in data collection and analysis allows presenting anything as significant. Psychological Science 22: 1359-66. [CrossRef]

Song, Fujian, Sheetal Parekh, Lee Hooper, Yoon K Loke, J Ryder, Alex J Sutton, C Hing, Chun Shing Kwok, Chun Pang, and Ian Harvey. 2010. Dissemination and publication of research findings: An updated review of related biases. Health Technology Assessment 14: 1-193. [CrossRef]

Spitz, Herman H. 1986. The Raising of Intelligence: A Selected History of Attempts to Raise Retarded Intelligence. Hillsdale: Lawrence Erlbaum Associates, Inc., Publishers.

Stanley, T. D., Evan C. Carter, and Hristos Doucouliagos. 2018. What meta-analyses reveal about the replicability of psychological research. Psychological Bulletin 144: 1325-46. [CrossRef]

Stern, Jerome M., and R. John Simes. 1997. Publication bias: Evidence of delayed publication in a cohort study of clinical research projects. British Medical Journal 315: 640-45. [CrossRef]

Sterne, Jonathan AC, Betsy Jane Becker, and Matthias Egger. 2005. The funnel plot. In Publication Bias in Meta-Analysis: Prevention, Assessment and Adjustments. Edited by Hannah R. Rothstein, Alexander J. Sutton and Michael Borenstein. New York: Wiley, pp. 75-98.

Sterne, Jonathan A.C., and Matthias Egger. 2005. Regression methods to detect publication and other bias in meta-analysis. In Publication Bias in Meta-Analysis: Prevention, Assessment and Adjustments. Edited by Hannah R. Rothstein, Alexander J. Sutton and Michael Borenstein. New York: Wiley, pp. 99-110.

Szucs, Denes, and John PA Ioannidis. 2017. Empirical assessment of published effect sizes and power in the recent cognitive neuroscience and psychology literature. PLoS Biology 15: e2000797. [CrossRef]

Trikalinos, Thomas A., and John PA Ioannidis. 2005. Assessing the evolution of effect sizes over time. In Publication Bias in Meta-analysis: Prevention, ASSESSMENT and Adjustments. Edited by H. R. Rothstein, A. J. Sutton and Michael Borenstein. New York: Wiley, pp. 241-59.

Van Aert, Robbie CM, Jelte M. Wicherts, and Marcel ALM Van Assen. 2019. Publication bias examined in meta-analyses from psychology and medicine: A meta-meta-analysis. PLoS ONE 14: e0215052. [CrossRef] [PubMed]

Van Assen, Marcel A.L.M., Robbie van Aert, and Jelte M. Wicherts. 2015. Meta-analysis using effect size distributions of only statistically significant studies. Psychological Methods 20: 293-309. [CrossRef] [PubMed]

Van Dalen, Hendrik P., and Kene Henkens. 2012. Intended and unintended consequences of a publish-or-perish culture: A worldwide survey. Journal of the American Society for Information Science and Technology 63: 1282-93. [CrossRef]

Van Der Maas, Han L.J., Conor V. Dolan, Raoul P.P.P. Grasman, Jelte M. Wicherts, Hilde M. Huizenga, and Maartje E.J. Raijmakers. 2006. A dynamical model of general intelligence: The positive manifold of intelligence by mutualism. Psychological Review 113: 842-61. [CrossRef]

Veroniki, Areti Angeliki, Dan Jackson, Wolfgang Viechtbauer, Ralf Bender, Jack Bowden, Guido Knapp, Oliver Kuss, Julian Higgins, Dean Langan, and Georgia Salanti. 2016. Methods to estimate the between-study variance and its uncertainty in meta-analysis. Research Synthesis Methods 7: 55-79. [CrossRef]

Vevea, Jack L., and Larry V. Hedges. 1995. A general linear model for estimating effect size in the presence of publication bias. Psychometrika 60: 419-35. [CrossRef] 
Viechtbauer, Wolfgang. 2010. The Metafor Package: A Meta-Analysis Package for R (Version 1.3-0). Available online: http://cran.r-project.org/web/packages/metafor/index.html (accessed on 25 September 2020).

Wagenmakers, Eric-Jan, Ruud Wetzels, Denny Borsboom, Han L.J. van der Maas, and Rogier A. Kievit. 2012. An agenda for purely confirmatory research. Perspectives on Psychological Science 7: 632-38. [CrossRef]

Wagenmakers, E. J., Titia Beek, Laura Dijkhoff, Quentin F. Gronau, A. Acosta, R. B. Adams Jr., D.N. Albohn, E.S. Allard, S.D. Benning, and E-M Blouin-Hudon. 2016. Registered Replication Report: Strack, Martin, \& Stepper (1988). Perspectives on Psychological Science 11: 917-28. [CrossRef]

Wicherts, Jelte. 2013. Science revolves around the data. Journal of Open Psychology Data 1: e1. [CrossRef]

Wicherts, Jelte M., Coosje L.S. Veldkamp, Hilde E.M. Augusteijn, Marjan Bakker, Robbie Van Aert, and Marcel A.L.M. Van Assen. 2016. Degrees of freedom in planning, running, analyzing, and reporting psychological studies: A checklist to avoid p-hacking. Frontiers in Psychology 7: 1832. [CrossRef]

Zhang, Jian-Ping, Katherine E. Burdick, Todd Lencz, and Anil K. Malhotra. 2010. Meta-analysis of genetic variation in DTNBP1 and general cognitive ability. Biological Psychiatry 68: 1126-33. [CrossRef] [PubMed]

(C) 2020 by the authors. Licensee MDPI, Basel, Switzerland. This article is an open access article distributed under the terms and conditions of the Creative Commons Attribution (CC BY) license (http://creativecommons.org/licenses/by/4.0/). 